Reithmann - Martiny

Internationales Vertragsrecht 



\title{
Internationales Vertragsrecht
}

Das internationale Privatrecht der Schuldverträge

\author{
Dr. Christoph Reithmann \\ Prof. em. Dr. Dieter Martiny
}

8. neu bearbeitete Auflage

2015

\section{ottoschmidt}

Linde 



\section{Bearbeiterverzeichnis}

Prof. Dr. Anatol Dutta, M. Jur. (Oxford)

Universität Regensburg

Prof. Dr. Robert Freitag, Maître en droit Universität Erlangen-Nürnberg

Prof. Dr. Stephan R. Göthel, LL.M.

Rechtsanwalt und Wirtschaftsmediator in Hamburg,

Professor an der BSP Business School Berlin,

Lehrbeauftragter an der Bucerius Law School Hamburg

Prof. Dr. Pascal Grolimund, LL.M. Advokat in Basel

Veronika Häuslschmid

Rechtsanwältin, VDMA - Verband Deutscher Maschinen- und Anlagenbau e.V., Frankfurt/Main

Prof. em. Dr. Rainer Hausmann

Universität Konstanz

Rechtsanwalt in München

Dr. Martin Hiestand

Ministerialrat im Bundesministerium der Justiz und für Verbraucherschutz, Berlin

Prof. Dr. Oliver L. Knöfel

Europa-Universität Viadrina Frankfurt/Oder

Prof. Dr. Peter Limmer

Notar in Würzburg, Honorarprofessor an der

Julius-Maximilians-Universität Würzburg

Prof. Dr. Peter Mankowski

Universität Hamburg

Prof. em. Dr. Dieter Martiny

Europa-Universität Viadrina Frankfurt/Oder

Prof. Dr. Eva Inés Obergfell

Humboldt-Universität zu Berlin

Dr. Christoph Reithmann

Notar a.D., Wolfratshausen

Prof. Dr. Anton K. Schnyder, LL.M. Universität Zürich

Prof. Dr. Michael Stürner, M. Jur. (Oxford) Universität Konstanz

Richter am OLG Karlsruhe

Prof. Dr. Reinhold Thode

Richter am Bundesgerichtshof a.D.,

Rechtsanwalt in Landau/Pfalz,

Honorarprofessor an der Universität Konstanz

unter redaktioneller Mitarbeit von

Verena Reithmann

Rechtsanwältin in Icking 
Bibliografische Information

der Deutschen Nationalbibliothek

Die Deutsche Nationalbibliothek verzeichnet diese

Publikation in der Deutschen Nationalbibliografie;

detaillierte bibliografische Daten sind im Internet

über http://dnb.d-nb.de abrufbar.

Verlag Dr. Otto Schmidt KG

Gustav-Heinemann-Ufer 58, 50968 Köln

Tel. 02 21/9 37 38-01, Fax 02 21/9 37 38-943

info@otto-schmidt.de

www.otto-schmidt.de

ISBN Otto Schmidt Verlag: 978-3-504-45155-4

ISBN Linde: 978-3-7073-3405-0

(C2015 by Verlag Dr. Otto Schmidt KG, Köln

Das Werk einschließlich aller seiner Teile ist urheberrechtlich geschützt. Jede Verwertung, die nicht ausdrücklich vom Urheberrechtsgesetz zugelassen ist, bedarf der vorherigen Zustimmung des Verlages. Das gilt insbesondere für Vervielfältigungen, Bearbeitungen, Übersetzungen, Mikroverfilmungen und die Einspeicherung und Verarbeitung in elektronischen Systemen.

Das verwendete Papier ist aus chlorfrei gebleichten Rohstoffen hergestellt, holz- und säurefrei, alterungsbeständig und umweltfreundlich.

Einbandgestaltung: Jan P. Lichtenford, Mettmann

Satz: WMTP, Birkenau

Druck und Verarbeitung: Kösel, Krugzell

Printed in Germany 